\title{
Experimental damage analysis of steels after exploitation loading
}

\author{
Z.L. Kowalewski ${ }^{1,2, a}$, J. Szelążek ${ }^{1}$, S. Mackiewicz ${ }^{1}$, and B. Augustyniak ${ }^{3}$ \\ ${ }^{1}$ Institute of Fundamental Technological Research, ul. Pawińskiego 5B, 02-106 Warsaw, Poland \\ ${ }^{2}$ Motor Transport Institute, ul. Jagiellońska 80, 03-301 Warsaw, Poland \\ ${ }^{3}$ Gdańsk University of Technology, ul. Narutowicza 11/12, 80-952 Gdańsk, Poland
}

\begin{abstract}
Development of creep damage at elevated temperatures and structural degradation due to plastic deformation at room temperature were assessed using destructive and non-destructive methods in steels commonly applied in power plants (40HNMA, 13HMF and P91). As destructive methods the standard tension tests were carried out after every kind of prestraining. Subsequently, an evolution of the selected tension parameters was taken into account for damage identification. In order to assess a damage development during the creep and plastic deformation the tests for the steels were interrupted for a range of the selected strain magnitudes. The ultrasonic and magnetic techniques were used as the non-destructive methods for damage evaluation. The experimental programme also contained microscopic observations.
\end{abstract}

\section{Introduction}

Engineering materials subjected to exploitation loadings change their mechanical properties. Depending on the working conditions the variations of some selected parameters of these materials may attain such magnitudes that their further exploitation is risky due to possible failures. Such situations are dangerous for the devices posing a major threat to environment and human security. Power plants are the typical examples. Figure 1 presents the results showing a drastic reduction of creep lifetime of the $13 \mathrm{HMF}$ steel used for pipeline subjected to the long time exploitation at elevated temperature $(813 \mathrm{~K})$ under internal pressure $(14 \mathrm{bars})$. To avoid an unpredictable catastrophic accidents a systematic monitoring must be carrying out.

(a)

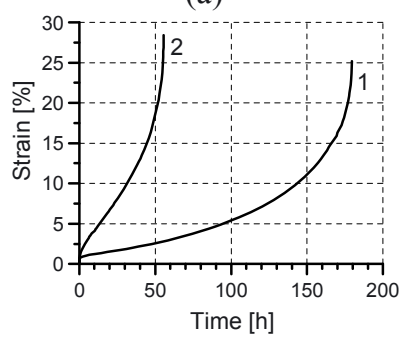

(b)

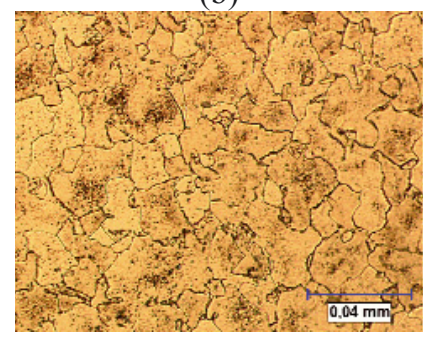

(c)

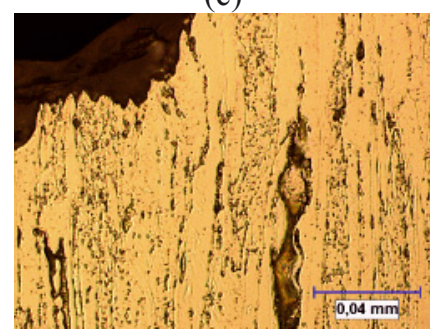

Fig. 1. Comparison of tensile creep curves $(\sigma=220 \mathrm{MPa}, \mathrm{T}=773 \mathrm{~K})$ for the $13 \mathrm{HMF}$ steel in the as-received state (1) and after exploitation within $76000 \mathrm{~h}(2)$ (a), and its metalographic structures for the initial state (b) and after $76000 \mathrm{~h}$ of work (c).

\footnotetext{
a e-mail : zkowalew@ippt.gov.pl
} 
There are many testing techniques commonly used for damage assessments. Among them we can generally distinguish destructive [1], and non-destructive methods [2]. Having the parameters of destructive and non-destructive methods for damage development evaluation it is worth to analyze their variation in order to find possible correlations.

The ultrasonic and magnetic techniques were selected as the non-destructive methods for damage development evaluation. In the case of ultrasonic method the acoustic birefringence coefficient was used to identify damage development in the tested steels. In the case of magnetic technique the classical Barkhausen effect (HBE) and magnetoacoustic emission (EMA) were measured. It is shown that both magnetic parameters are sensitive on the level of material damage.

\section{Procedures, results and discussion}

Uniaxial tension creep tests were carried out using plane specimens. For each steel all tests were conducted in the same conditions. In the case of 40HNMA and 13HMF the stress level was equal to $250 \mathrm{MPa}$, and temperature $-773 \mathrm{~K}$, whereas for P91 - $290 \mathrm{MPa}$, and $773 \mathrm{~K}$, respectively. In order to assess a damage development during the process of creep the tests were interrupted for a range of the selected time periods, which correspond to the increasing amounts of creep strain. In the case of 40HNMA and P91 steels some selected magnitudes of deformation were also applied to prestrain specimens by means of plastic flow at room temperature.

After prestraining the ultrasonic and magnetic investigations were carried out to identify a damage development in the tested steels. In the next step of the experimental procedure, the same specimens were mounted on a hydraulic servo-controlled MTS testing machine and then stretched until failure was achieved. The standard tensile tests were performed in order to evaluate variation of typical tensile parameters, i.e. Young's modulus, yield point, ultimate tensile stress. The last step of the experimental programme contains microscopic observation using optical and scanning microscopes.

\subsection{Evaluation of damage development using destructive tests}

In order to assess damage development during creep the tests for the 40HNMA steel were interrupted after 100h, 241h, 360h, 452h, 550h, 792h, 929h and 988h, which correspond to increasing amounts of creep strain from $0.34 \%$ up to $6.5 \%$. In the case of P91 the tests were interrupted after 40h, 180h, 310h, 390h, 425h, 440h and 445h (strain range $0.85 \%-9.3 \%$ ).

The tensile characteristics for the tested materials after prestraining are presented in Fig. 2a for the 40HNMA steel and in Fig. 2b for the P91 steel. In all of these diagrams the characteristics for the prestrained steel are compared with the tensile curve of the steels in the as-received state.

On the basis of these tensile characteristics, Fig. 2, variations of the basic mechanical properties of both steels, due to deformation achieved by prior creep or plastic flow were determined. It was observed for both materials, that the Young's modulus is almost insensitive to the magnitude of creep and plastic deformations. Contrary to the Young's modulus the other considered tension test parameters, especially the yield point and the ultimate tensile stress, Figs. 3 and 4, exhibit clear dependence on the level of prestraining.

Taking into account the results presented for the 40HNMA steel in Figs. 2a and 3 it is easy to note that this material exhibits a significant softening effect due to the creep prestraining, expressed by a large decrease of the yield point and ultimate tensile stress. An opposite effect can be observed for this material prestrained due to plastic deformation at room temperature. In this case the prior deformation leads to a hardening effect.

Similar results were obtained for the P91 steel. In this case, however, differences between magnitudes of such parameters as the yield point or ultimate tensile stress observed for the same value of prior deformation induced by creep and plastic flow are not so large as those for the 40HNMA steel. Again, prior plastic deformation caused the hardening of the steel, while creep prestraining led to its softening. It is important to note that the observed softening effect is only 
expressed on the basis of the ultimate tensile stress variations since for the testing conditions applied in these investigations the magnitude of the yield point is not sensitive to the amount of prior creep deformation. More details of investigations of the 40HNMA and P91 steels are described in [3, 4]

(a)

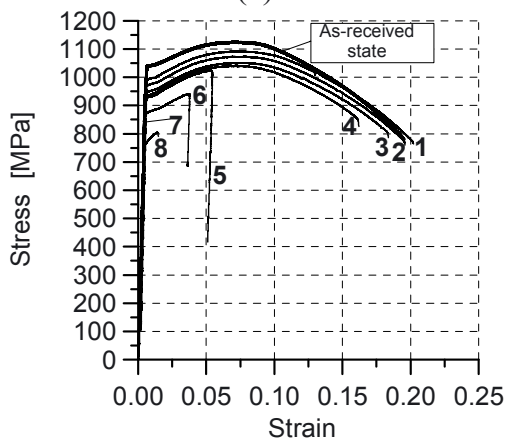

(b)

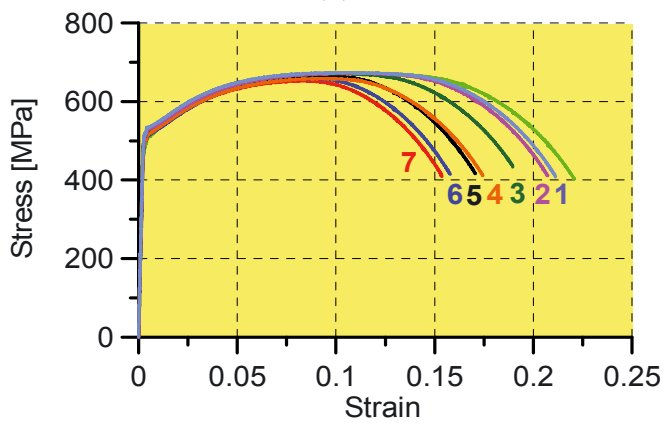

Fig.2. Tension characteristics of: (a) the 40HNMA steel after creep for 100h (1), 241h (2), 360h (3), 452h (4), 550h (5), 792h (6), 929h (7) and 988h (8), and (b) the P91 steel after creep for 40h (1), 180h (2), 310h (3), 390h (4), 425h (5), 440h (6) and 445h (7). Non-numbered curves correspond to the steels in the as-received state.

(a)

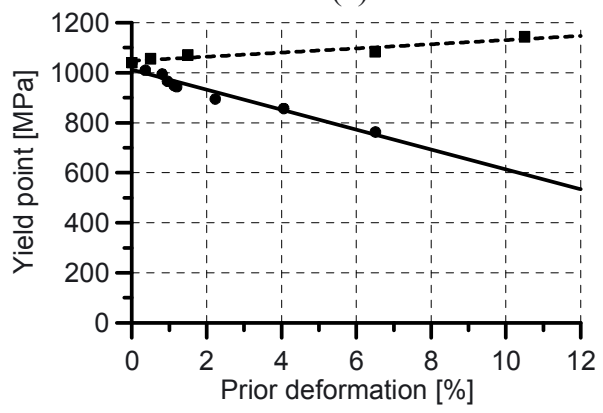

(b)

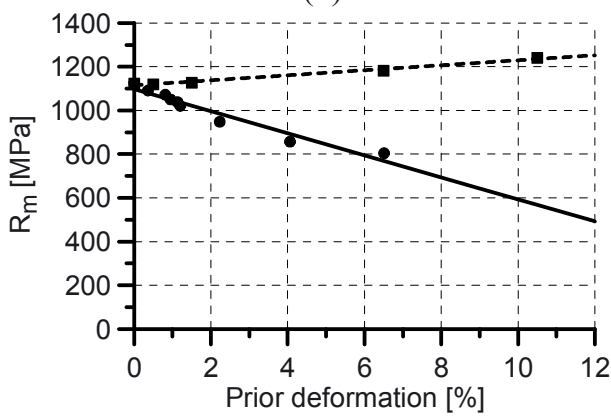

Fig. 3. Variation of the tensile parameters for the 40HNMA steel due to creep (solid lines) and plastic (broken lines) deformations: (a) yield point; (b) ultimate tensile stress.

(a)

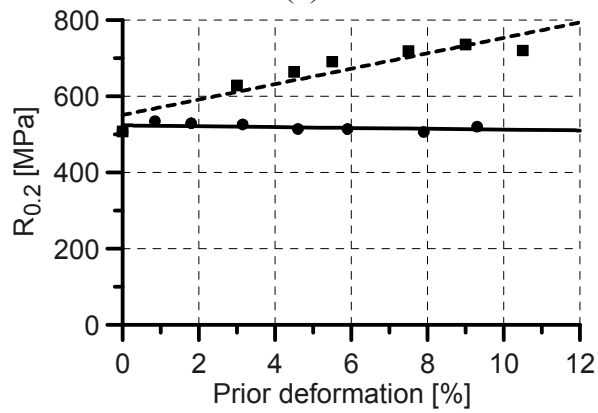

(b)

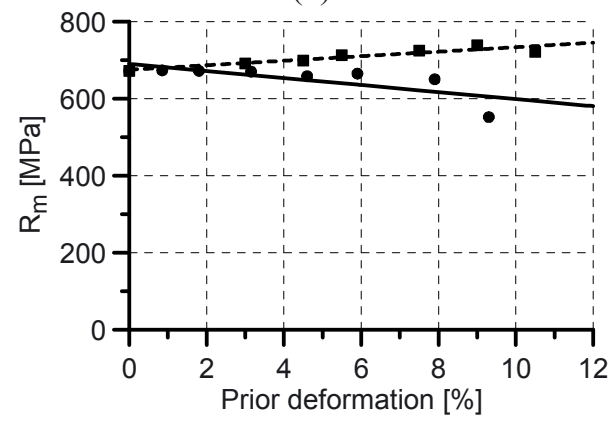

Fig. 4. Variation of tensile parameters of the P91 steel due to creep (solid lines) and plastic (broken lines) deformations: (a) yield point; (b) ultimate tensile stress. 
The tensile characteristics for the 13HMF steel after creep prestraining are presented in Fig. 5. It is easy to notice that the results are different then those for the 40HNMA and P91 steels obtained. The material exhibits a significant hardening effect due to the creep prestraining, expressed by an increase of the yield point, Fig. 5, and ultimate tensile stress, Fig. 6.

(a)

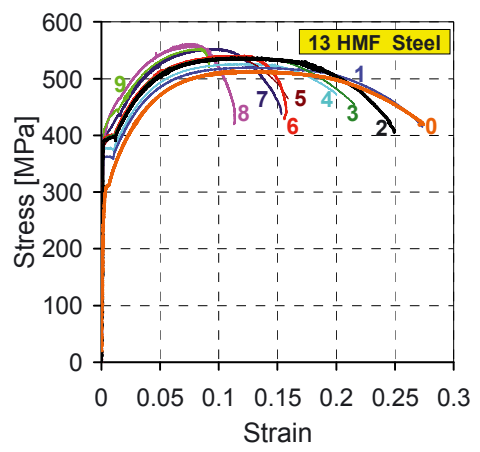

Fig. 5. Tension characteristics of the $13 \mathrm{HMF}$ steel after creep for $149 \mathrm{~h}(1), 300 \mathrm{~h} \mathrm{(2),360h} \mathrm{(3),} \mathrm{407h} \mathrm{(4),}$ 441h (5), 587h (6), 664h (7), 796h (8) and 1720h (9). (b)

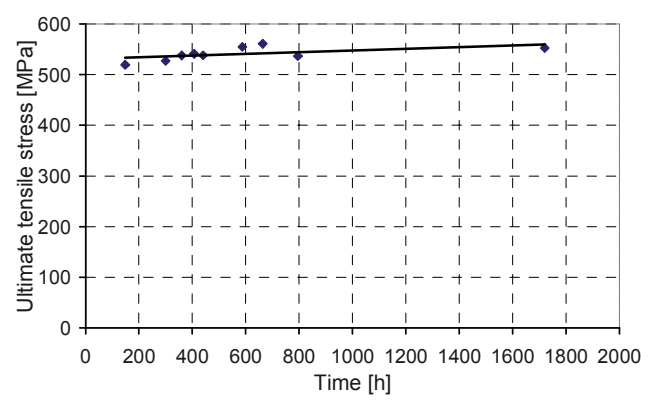

Fig. 6. Variation of the ultimate tensile stress due to prior deformation by creep for the 13HMF steel.

\subsection{Evaluation of damage development using ultrasonic technique}

Figure 7 presents mean values of the acoustic birefringence measured in specimens after creep or plastic deformation. The birefringence was measured in the fixtures, where the texture of the material was assumed to be unchanged during creep testing, and in the working part of the specimen. Values of birefringence measured in the fixture exhibit some scatter around zero, Fig.7a. This scatter is a picture of birefringence evaluation accuracy and the initial acoustic homogeneity of the specimen. In the deformed part of specimen the birefringence depends on the amount of deformation. It can be noticed that birefringence variations due to creep are significantly higher than birefringence scatter for the non-deformed material. The acoustic birefringence was measured at several points along the working part of each specimen, thus enabling its maximum to be found. For the maximum creep prestrained specimen, where the necking was visible, the birefringence maximum was measured in the specimen neck. For less deformed specimens, in which necking was not observed, one can expect that the birefringence maximum indicates the region of maximum micro defect concentration. These regions can be treated as the sources of future macro defects leading finally to failure. The plots presented in Fig. 7 indicate that the acoustic birefringence is sensitive to the amount of prior deformation. Another advantage of this parameter is also well represented in Fig. 7. Namely, it is very sensitive to the form of prior deformation. This feature is especially well revealed in the case of the birefringence determined for the 40HNMA steel, Fig. 7a. For specimens prestrained due to creep the increase of this parameter is observed with the increase of prior deformation. An opposite effect was achieved for specimens prestrained due to the plastic deformation at room temperature, i.e. with the increase of prior deformation a decrease of the birefringence was obtained. Also, the results for P91 steel allow the type of prior deformation to be identified, however, the effect is not so clear as for the 40HNMA steel.

The results show that the acoustic birefringence can be a quite sensitive indicator of material degradation and can help to locate the regions where material properties are changed due to creep. Measurements of the ultrasonic wave attenuation and velocities carried out on the same steels did not exhibit such a good sensitivity in the material damage assessments.

More details concerning the results and testing technique are available in [5-7]. 
(a)

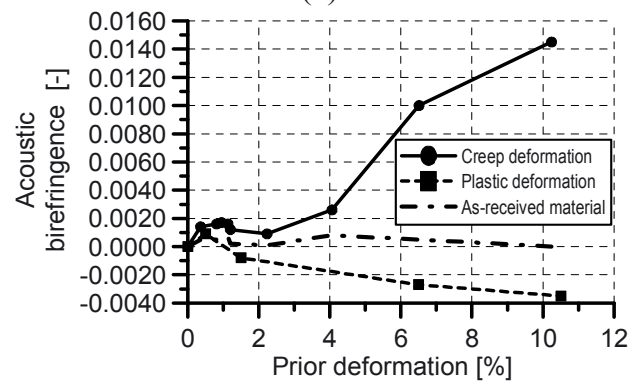

(b)

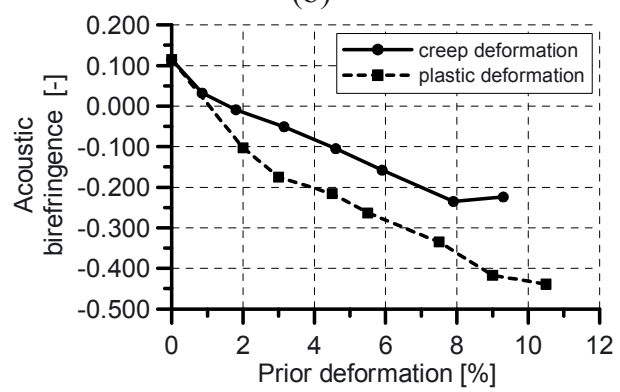

Fig. 7. Acoustic birefringence B variations due to prior deformation for: (a) 40HNMA steel, (b) P91 steel.

\subsection{Evaluation of damage development using magnetic techniques}

Two magnetic techniques for non-destructive testing were applied, i.e. measurement of Barkhausen effect (HBE) and magneto-acoustic emission (EMA) [2, 8, 9]. Both effects are due to abrupt movement of magnetic domain walls depicted from microstructural defects when sample is magnetised. The samples at laboratory tests were magnetised by the solenoid and a magnetic flux generated in the sample was closed by $\mathrm{C}$-core like shaped yoke. Magnetizing current (delivered by current source) had a triangular like waveform and frequency of order $0.1 \mathrm{~Hz}$. Its intensity was proportional to the voltage $U g$. Two sensors were used: (a) the pickup coil (PC), and (b) the acoustic emission transducer (AET). Voltage signal induced at PC was used for magnetic hysteresis loop $B(H)$ evaluation (low frequency component) as well as for $\mathrm{HBE}$ analysis (high frequency component). Intensity of $\mathrm{HBE}$ is given by rms (root mean square) voltage $U b$ envelopes. In this case the maximum ( $U b p p)$ of $U b$ for one period of magnetisation is compared. Analogue analysis is performed for MAE voltage signal from the AET. The magnetic coercivity $H c$, evaluated from the $B(H)$ hysteresis loop plots, is also compared. An influence of plastic flow and creep damage on the basic magnetic properties can be analysed using $B(H)$ hysteresis loops. The representative results showing variations of the $B(H)$ hysteresis loops are presented in Figs. 8 and 9 for the P91 steel. The curves obtained for an undamaged specimen $(\varepsilon=0 \%)$ and for all the damaged ones are compared. The quantity $U g$ denotes the voltage proportional to the driving current intensity, and hence magnetic field strength $H$. The as described features of the HBE intensity are well presented by means of plots showing a dependence between the amplitudes of $U b$ envelopes and magnitudes of prior deformation - peak to peak values $U b_{p p}$ in Fig. 10. Thus, one can say that the HBE intensity as a function of the resulting prestrain peaks firstly and then decreases monotonicaly when amplitudes of the $U b$ envelopes are compared. The curves in Fig. 10 reveal also that creep damage leads (at its final stage) to a greater 'decrease' of the HBE intensity than that observed for specimens after plastic flow. Comparing two plots in the figure it can be seen that the $U b$ signal properties such as the amplitude for the highest strain after creep damage are roughly the same as for the analogous signals for the first stage of plastic flow.

A synthetic description of the MAE properties as a function of prior deformation is given by plots shown in Fig. 11 (amplitudes of the MAE envelopes). Amplitudes of the MAE intensity decrease for both cases but the dynamics of their change is different. Moreover, amplitudes of the MAE intensity envelopes do not decrease so abruptly for the creep prestrained specimens, and do not reach the level obtained for the first step of plastic deformation due to plastic flow at room temperature.

The representative results showing variations of the $B(H)$ hysteresis loops are also obtained for the 13HMF steel prestrained due to creep, Fig. 12. Comparison of the results for P91 (Fig. 9) and 13HMF (Fig.12) steels identifies different tendency of the magnetic hysteresis loops variation. This is in agreement with a variation of the mechanical parameters achieved for these materials. 


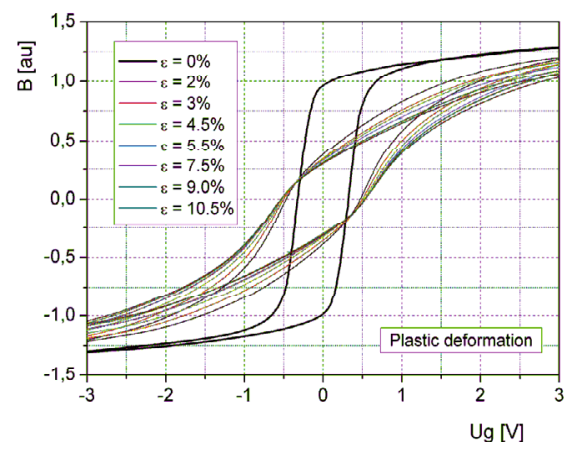

Fig. 8. Magnetic hysteresis loops of undamaged $(\varepsilon=0)$ and damaged specimens due to plastic flow.

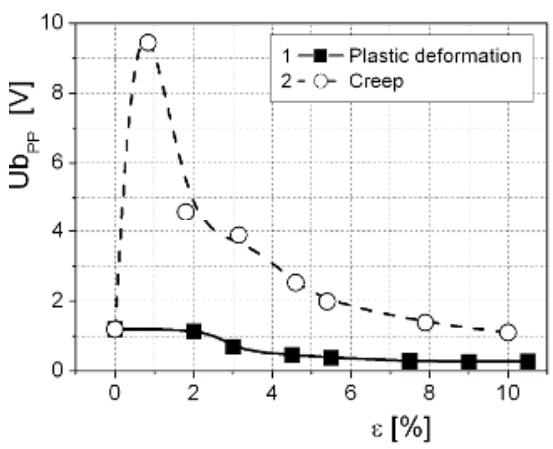

Fig. 10. Dependence between the amplitudes of Ub envelopes and deformation for specimens after plastic flow (squares) and after creep (circles).

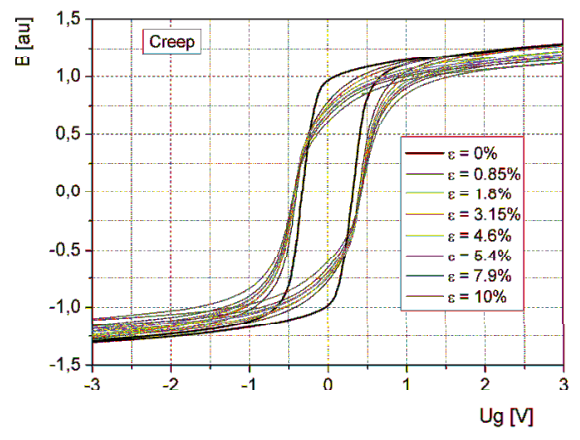

Fig. 9. Magnetic hysteresis loops of undamaged $(\varepsilon=0)$ and damaged specimens due to creep.

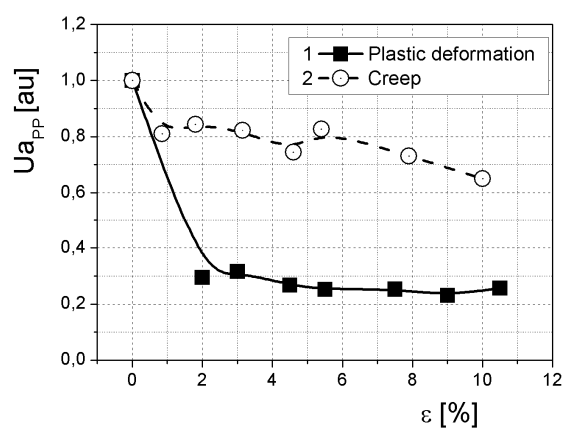

Fig. 11. Dependence between amplitudes of Ua envelopes and prior deformation due to plastic flow (squares) and creep (circles).

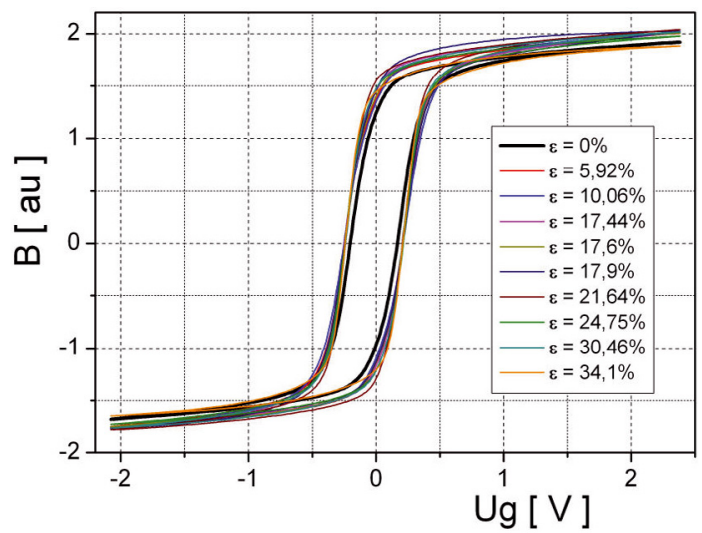

Fig. 12. Magnetic hysteresis loops of undamaged $(\varepsilon=0)$ and damaged specimens due to creep of the 13HMF steel.

\subsection{Identification of damage using metallographic observations}

The last step of the experimental programme contained microscopic observations. The metallographic assessments were carried out by means of optical microscope (Olympus PMG3 - in macro- and micro- ranges) as well as by means of scanning electron microscopy (SEM - JEOL 6360 
LA) techniques [10]. All observations were done in non-etched and etched state. The effect of voids formation was observed along the longitudinal metallographic sections prepared from specimens after completion of the mechanical tests. Then, the selected geometrical parameters of existing voids were determined by means of the image analysis in the optical microscopy range. The following parameters were determined: depth of void $[\mathrm{mm}]$, mean area fraction of voids $\left(\mathrm{A}_{\mathrm{A}}[\%]\right)$ related to the unit area of metallographic sample $\left(1 \mathrm{~mm}^{2}\right)$ and mean quantity of voids $-\mathrm{N}_{\mathrm{A}}\left[1 / \mathrm{mm}^{2}\right]$, Fig. $13 \mathrm{~b}$.

The comparison of microstructural effects in the 40HNMA steel and determined geometrical parameters show the greatest damage at test 8 (Fig.2), Fig.13. It is characteristic that the voids dimensions are bigger in the perpendicular direction with respect to the specimen axis than those observed parallel. For lower prior creep deformations the damage was connected rather with the nonmetallic inclusions. In all these cases the fragmentations of existing nonmetallic inclusions, and subsequently, voids formation were observed. The microstructure for all specimens was the same, i.e. sorbite with remaining the needle martensite configuration.

(a)

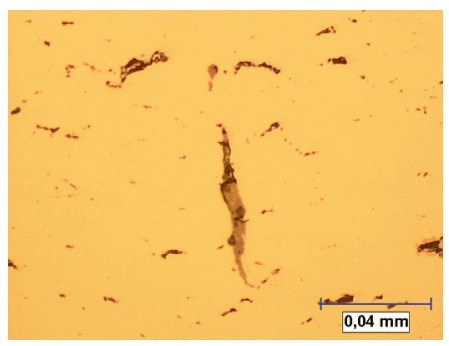

(b)

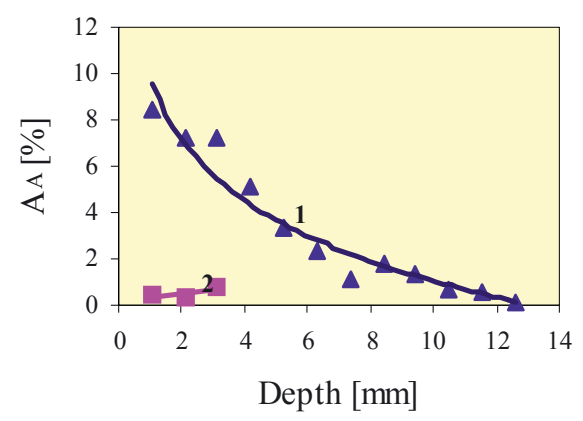

Fig. 13. Microstructural observations of the 40HNMA: (a) strong damage close to fracture surface, magnification 500x; (b) changes of the void area fractions $\left(\mathrm{A}_{\mathrm{A}}[\%]\right)$ as a function of distance from the fracture surface for 40HNMA steel deformed due to: (1) creep up to $6.5 \%$; (2) plastic flow up to fracture (19\%).

The effects observed in the case of P91 steel are generally different than those for the 40HNMA steel observed despite use of almost the same testing conditions. In the case of the creep prestrained material it is possible to observe cracks, located close to the fracture surface with lengths from 0,0046 to $0,1416 \mathrm{~mm}$ and directed perpendicularly to the fracture surface. For plastic prestrained specimens, a half of them (for smaller magnitudes of deformation) demonstrate fractures without well-marked cracks. In the case of higher amounts of prior plastic deformation some small cracks are visible, and they are located perpendicular to the specimen fracture surface. Contrary to the 40HNMA, the P91 steel, Fig. 14, contains very few nonmetallic inclusions (as potential structural notches).

(a)

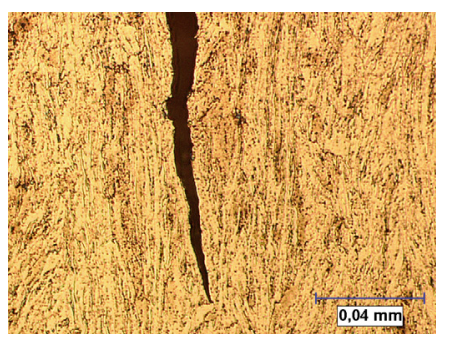

(b)

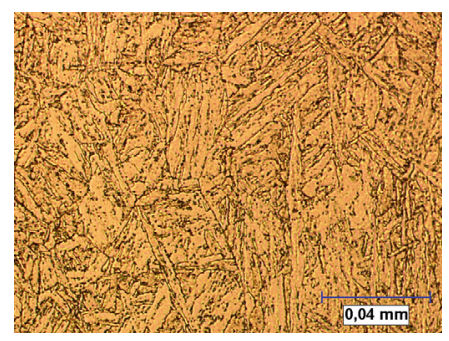

Fig. 14. Microstructure of the P91 steel in the etched state, 500x: (a) close to fracture surface; (b) far from fracture surface. 
Therefore, the microstructure is deformed by grain elongation up to the loss of the microstructural connections affecting crack formation near the fracture surface only. The microstructure observed relatively far from the fracture surface is not mechanically deformed and consists of the ferrite and carbides mixture with the remaining needle-like martensite configuration, Fig. 14b.

\section{Conclusions}

The paper presents the results of interdisciplinary tests for damage assessments in steels commonly used for pipelines at power stations.

The results show that ultrasonic and magnetic parameters can be good indicators of material degradation and can help to indicate the regions where material properties are changed due to prestraining.

In the case of ultrasonic investigations the results show that the attenuation of ultrasonic waves is in practice stable until the final stages of creep. It was also observed that the velocity changes due to creep or plastic deformation are small, and therefore, an application of the velocity measurement for damage evaluation in industrial conditions is very difficult. In order to evaluate damage progress in specimens made of the 40HNMA and P91 steels, instead of velocity and attenuation measurement, the acoustic birefringence $B$ measurements were succesfully applied.

In the case of magnetic investigations for damage identification the measurements of the Barkhausen effect (HBE) and the magneto-acoustic emission (MAE) were applied. Both effects show that the magnetic properties are highly influenced by prior deformation, and moreover, they are sensitive not only to the magnitude of prior deformation, but also to the way it is introduced.

\section{Acknowledgement}

The support from the National Centre for Research and Development (Poland) under grant NR 15$0049-04 / 2008$ is greatly appreciated.

\section{References}

1. ASTM Handbook Vol. 19, Fatigue and Fracture, ASM International (1996)

2. M.J. Sablik, B. Augustyniak, in Wiley Encyclopedia of Electrical and Electronics Engineering, ed. J.G. Webster, (J. Wiley\&Sons, New York, 1999)

3. Z.L. Kowalewski, J. Szelążek, S. Mackiewicz, K. Pietrzak, B. Augustyniak, International Journal of Modern Physics Letter B, 22, No 31/32, 5533 (2008)

4. Z.L. Kowalewski, J. Szelążek, S. Mackiewicz, K. Pietrzak, B. Augustyniak, Journal of Multiscale Modeling, 1, No 3\&4, 479 (2009)

5. Z.L. Kowalewski, S. Mackiewicz, J. Szelążek, J. Deputat, in Proc. of XXI Symp. on Experimental Mechanics of Solid Body, ed. J. Stupnicki (Warsaw University of Technology, 2004)

6. S. Mackiewicz, Z.L. Kowalewski, J. Szelążek, J. Deputat, Mechanical Review, 7/8, 15 (2005)

7. J. Szelążek, S. Mackiewicz, Z.L. Kowalewski, NDT and E International, 42, 150 (2009)

8. B. Augustyniak, M. Chmielewski, M.J. Sablik, IEEE-Trans. on Magnetics, 36, 5, 3624 (2000)

9. B. Augustyniak, M. Chmielewski, L. Piotrowski, Z. Kowalewski, IEEE Transactions on Magnetics, 4, no 11, 3273 (2008)

10. K. Pietrzak, Z.L. Kowalewski, D. Rudnik, A. Wojciechowski, in Proc. of the XIV International Symposium on Plasticity and Its Current Applications, ed. A. Khan (NEC Press, Baltimore, 2008) 